\title{
Topology optimization for vibrating structures with the BESO method
}

\author{
Christian Brecher ${ }^{1}$, Guifeng Zhao ${ }^{2}$, Marcel Fey ${ }^{3}$ \\ Laboratory for Machine Tools and Production Engineering, Aachen, Germany \\ ${ }^{2}$ Corresponding author \\ E-mail: ${ }^{1}$ C.Brecher@wzl.rwth-aachen.de, ${ }^{2}$ G.Zhao@wzl.rwth-aachen.de, ${ }^{3}$ M.Fey@wzl.rwth-aachen.de
}

Received 18 March 2019; accepted 25 March 2019

DOI https://doi.org/10.21595/vp.2019.20673

Check for updates

Copyright $(\underset{C}{ } 2019$ Christian Brecher, et al. This is an open access article distributed under the Creative Commons Attribution License, which permits unrestricted use, distribution, and reproduction in any medium, provided the original work is properly cited.

\begin{abstract}
There is a lot of literature concerning the topology optimization of structures under static loads with the bi-directional evolutionary structural optimization (BESO), but only few approaches has focus on the dynamics problems with the BESO. This paper presents the von Mises stress as a sensitivity number and a new filter scheme for the BESO method focusing on dynamics problems. Based on the proposed technique and the BESO method, we discuss two measures to reduce vibrations of structures subjected to single external harmonic loads at a user-defined point. The natural frequency-based measure (NFBM) shifts the natural frequency of the most significant mode away from the driving frequency, while the steady state dynamics-based measure (SSDBM) considers several modes and natural frequencies at the same time using modal superposition.
\end{abstract}

Keywords: structural dynamics, topology optimization, BESO, von Mises stress, steady state dynamics.

\section{Introduction}

Structural optimization seeks to achieve the best performance for a structure while satisfying various constraints such as a given amount of material [1]. In recent decades, structural optimization methods have gained great progress with the increasing performance of computers and computing algorithms [2]. Structural optimization methods can be classified into three categories: sizing optimization, shape optimization and topology optimization [3]. Compared with other types of structural optimization, topology optimization of continuum structures is by far the most challenging technically [1]. Different types of topology optimization methods exist. As an important subtype, the evolutionary structural optimization (ESO) method was initially proposed by Xie and Steven based on a simple concept that a structure evolves towards an optimum by gradually removing less stressed material [4]. Yang expanded the ESO method to bi-directional evolutionary structural optimization (BESO) method allowing the recovery of the deleted elements which are neighboring to highly stressed elements [5]. In 2007, Huang and Xie improved the BESO method with a sensitivity filter scheme and a stabilization scheme using the history information [6], which is the latest major update for the BESO method. The latest version of BESO method includes the following procedure [6]: 1) Discretize the design domain using a finite element mesh; 2) Perform finite element analysis and then calculate the smoothed elemental sensitivity number; 3) Average the sensitivity number with its history information; 4) Determine the target volume for the next iteration; 5) Add and delete elements according to the sensitivity number; 6) Repeat steps 2-5 until the constraint volume is achieved.

The BESO method has been used in several static optimization problems, but it is not widely used in dynamics problems. Possible reasons for this are the computation complexity and the possible deletion of load regions.

In this paper, we propose the von Mises stress as a sensitivity number and a new filter scheme for the BESO method focusing on dynamics problems, and use it with a commercial Finite Element Analysis (FEA) software to implement the dynamics topology optimization. We will use the presented methods for the large-scale 3D topology optimization for machine tools in the future. 
The organization of the paper is as follows: in Section 2, we define the use case and propose two vibration reduction measures. In Section 3, we clarify the selection of the sensitivity number for the BESO method and the new filter scheme. Based on Section 2 and Section 3, we implement the two measures and present one numerical example in Section 4 and 5. In Section 6, we give a brief comparison between proposed BESO methods in this paper and existing evolutionary methods (ESO and BESO). We present the conclusions from this study in Section 7.

\section{Dynamics optimization problem under a single external harmonic loading}

In many industrial use cases, especially in the machine tool industry engineers want to reduce the vibration amplitude at a user-defined point. This paper discuss two measures to achieve this goal for structures under a single external harmonic loading. The NFBM shifts the natural frequency of the most significant mode away from the driving frequency, while the SSDBM consider several modes and natural frequencies at the same time using modal superposition.

\section{Sensitivity number and filter scheme}

The basic idea of the BESO method is to remove the unnecessary material from the structure. A reliable indicator of the inefficient use of material is a low value of stress in some parts of the structure. Nowadays, the distortion energy theory for ductile material uses the von Mises stress as an equivalent or effective stress to indicate the stress level of a continuum element within a structure under the three-dimensional loading [7]:

$\sigma^{\prime}=\frac{1}{\sqrt{2}}\left[\left(\sigma_{1}-\sigma_{2}\right)^{2}+\left(\sigma_{1}-\sigma_{3}\right)^{2}+\left(\sigma_{2}-\sigma_{3}\right)^{2}\right]^{1 / 2}$

The von Mises stress has been applied with the BESO method for static problems [8]. In this paper, the von Mises stress is used with the BESO method to indicate the inefficient use of material under dynamic loading.

Most of all BESO is a hard kill method, which means one element can only be solid or void and not have intermediate densities. This property is one of the most important advantages of the BESO method, which saves much computation time. But it can also cause a serious problem: sometimes it removes the elements, which transmit external loads into the structure. In order to avoid this problem, we propose a new filter scheme for the sensitivity number:

$\bar{\sigma}_{s i}^{\prime}=\frac{\sigma_{s i}^{\prime}}{\sigma_{s_{-} \max }^{\prime}}, \quad \bar{\sigma}_{d i}^{\prime}=\frac{\sigma_{d i}^{\prime}}{\sigma_{d_{-} \max }^{\prime}}, \quad \alpha_{i}=\frac{w * \bar{\sigma}_{s i}^{\prime}+(1-w) * \bar{\sigma}_{d i}^{\prime}}{r_{i}^{p}}$,

where $\sigma_{s i}^{\prime}, \sigma_{s_{-} \max }^{\prime}$ and $\bar{\sigma}_{s i}^{\prime}$ are the von Mises stress for the $i$ th element, the maximum of von Mises stress, and the normalized von Mises stress for the $i$ th element in static simulation. $\sigma_{d i}^{\prime}, \sigma_{d}^{\prime} \max$ and $\bar{\sigma}_{d i}^{\prime}$ are the von Mises stress for the $i$ th element, the maximum of von Mises stress and the normalized von Mises stress for the $i$ th element in dynamics simulation. $\alpha_{i}$ is the sensitivity number for $i$ th element, $w$ is the weighting factor of the static simulation, $1-w$ is the weighting factor of the dynamics simulation, $r_{i}$ is the distance between centroid of the $i$ th element and the load point, and $p$ is the distance penalty factor. The influence of the distance $r_{i}$ increases with the $p$, where $p$ is not smaller than 0 . This filter scheme can be expanded to multiple loading points.

We take the dynamics analysis, static analysis and the position of the load point into account with this filter scheme. The influences of the static analysis and the position of the load point depend on the weighting factor $w$ and the distance penalty factor $p$. 


\section{Vibration reduction measures}

\subsection{Natural frequency based measure (NFBM)}

In this subsection, we discuss how to push the most significant natural frequency away from the driving frequency with the BESO method.

Because the BESO method has a standard procedure as mentioned in the introduction, here we only clarify our implementation of step 2 , the other steps are same as the standard procedure. In step 2 of the BESO method, we perform the FEM analysis, which includes the static stress simulation, the natural frequency extraction simulation and the steady state dynamics simulation. We get the static von Mises stress from the static stress simulation. As a result of the natural frequency extraction simulation we get the natural frequency and the dynamic von Mises stress of all modes, then we use the static von Mises stress and dynamic von Mises stress of the most significant mode to calculate the sensitivity number with the filter scheme proposed in Section 3 . Thereafter we smooth the filtered sensitivity number again with a mesh-dependency and checkerboard filter proposed in paper von Xie and Huang [6].

The natural frequencies of the structure change after every iteration step, therefore the order of the modes could also vary, this phenomenon is called mode switching. In order to avoid the influence of the mode switching and track the most significant mode after every iteration step, we employ a mode tracking technique using a so-called Modal Assurance Criterion (MAC) [9]:

$M A C=\frac{\left|\left\{\varphi_{r}\right\}^{T}\left\{\varphi_{x}\right\}\right|^{2}}{\left(\left\{\varphi_{r}\right\}^{T}\left\{\varphi_{r}\right\}\right)\left(\left\{\varphi_{x}\right\}^{T}\left\{\varphi_{x}\right\}\right)}$,

where $\left\{\varphi_{r}\right\}$ is the reference eigen vector and $\left\{\varphi_{x}\right\}$ is the test eigen vector. The MAC takes values between 0 (representing no consistent correspondence) and 1 (representing a consistent correspondence).

We save the eigen vector of the most significant natural frequency after each iteration and use it as the reference eigen vector $\left\{\varphi_{r}\right\}$ in the next iteration, the eigen vectors after the new iteration are the test eigen vectors $\left\{\varphi_{x}\right\}$. We calculate MAC for all the test eigen vectors, and the test eigen vector with the maximum of MAC values is used as the new reference vector, the associated mode being the target mode for the optimization.

\subsection{Steady state dynamics-based measure (SSDBM)}

The most important difference between the SSDBM and the NFBM is how the dynamic von Mises stress is derived. In step 2 of the BESO method, the NFBM uses the dynamic von Mises stress in the natural frequency extraction simulation as the dynamic sensitivity indicator. Here, we use the dynamic von Mises stress in the steady state dynamics simulation as the dynamic sensitivity indicator.

Because the SSDBM concerns about the superposition of all the calculated modes, we do not need the MAC mode tracking technique.

\section{Numerical example}

We present one numerical example to show the effect of those two measures. The example is a traditional 3D cantilever beam as shown in Fig. 1. The external harmonic load excites the beam at the middle point of the right edge with a driving frequency of $7500 \mathrm{~Hz}$. The target volume is $70 \%$ of the design domain. The objective is to minimize the vibration amplitude in $Y$-direction at the load point. We calculate the vibration amplitude at the load point in the mode-based steady state dynamics analysis, which considers the first 10 modes in this example. We set the above proposed weighting factor of the static simulation $w$ as 0.1 and the distance penalty factor $\mathrm{p}$ as 0.5 
(see Eq. (2)).

The first four modes before the optimization process are shown in Fig. 1.
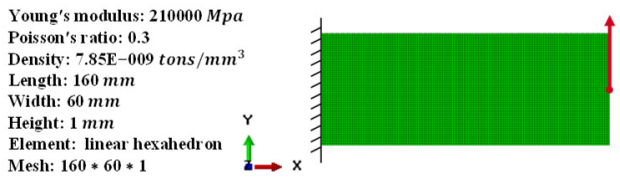

Fig. 1. Design problem for the example

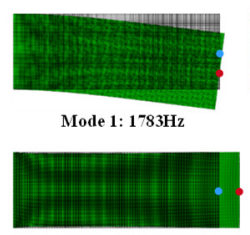

Mode 3: $8111 \mathrm{~Hz}$

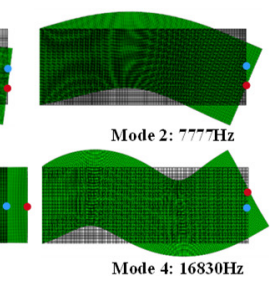

\subsection{Vibration reduction with the NFBM}

Mode 2 and 3 are both significant modes, because their natural frequencies are near the driving frequency. In order to pick the most significant one, we observe these two mode shapes as shown in Fig. 1. The displacement of load point in mode 2 is mainly in $Y$-direction whereas in mode 3 is mainly in $X$-direction. Because we only consider the vibration in $Y$-direction in our example, the mode 2 is most relevant.

Fig. 2 shows the optimization history with the NFBM. In the first ten iterations, the displacement at load point reduces obviously, because the natural frequency of mode 2 increases and therefore moves away from the driving frequency. The second natural frequency still increases after 10 iterations, but the fourth natural frequency decreases dramatically, the displacement of the load point in mode 4 is also mainly in $Y$-direction, so the vibration reduction effect is compensated. The displacement starts to increase after 16 iterations, which means the total influence of other 9 modes has suppressed the influence of the second mode. As shown in (d) of Fig. 2, the mode 2 moves away from the driving frequency, while another significant mode moves closer. The most significant mode could change after the turnover point, which is shown in (b) of Fig. 2.

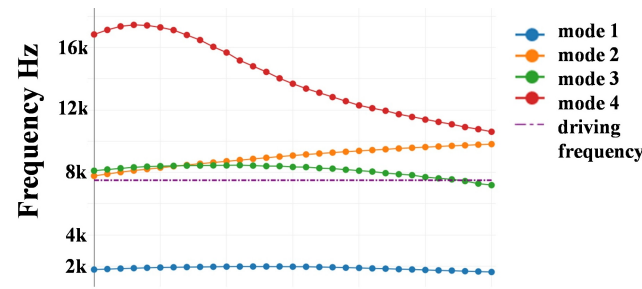

a)

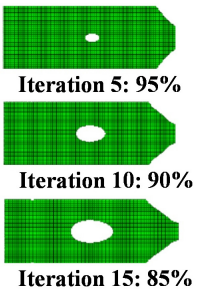

c)

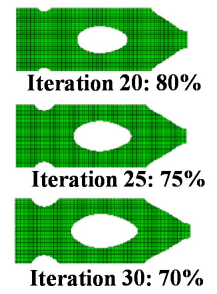

Iteration 30: 70\%

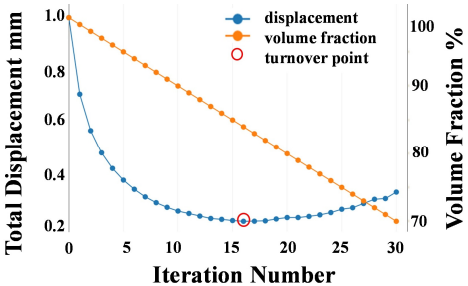

b)

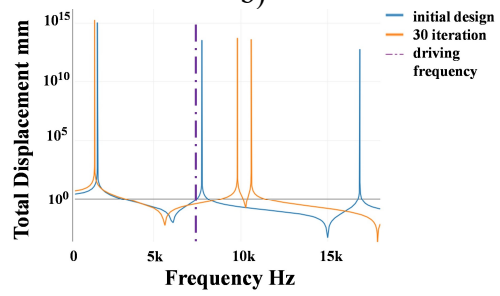

d)

Fig. 2. Optimization history: a) frequency, b) displacement, c) topology history, d) FRF

\subsection{Vibration reduction with the SSDBM}

Fig. 3 shows the optimization history with the SSDBM. We can see clearly, the displacement at load point keeps decreasing for 30 iterations. Because this measure considers not only the most significant mode 2, but also other 9 modes. As shown in Fig. 3, the second natural frequency 
increases more slowly as in the NFBM, but mode 1 is pushed away from the driving frequency and the mode 4 approaches the driving frequency slower than with the NFBM. The synergy effect of the changes of all natural frequencies contribute to the continuous minimization of the vibration at the load point.

The vibration reduction slows with the increasing iteration number. The reason for this is: one can't reduce the vibration and the amount of structure material at the same time with no limit.

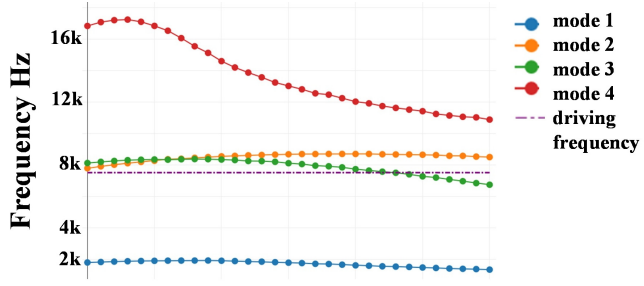

a)
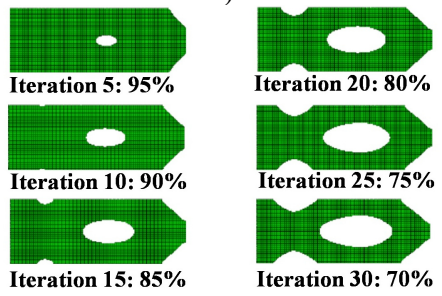

c)

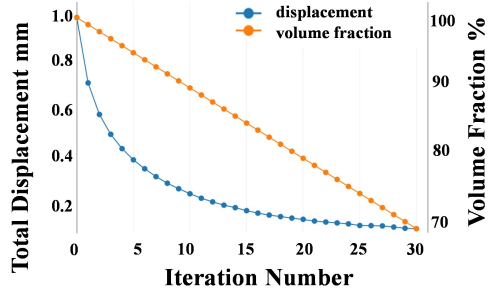

b)

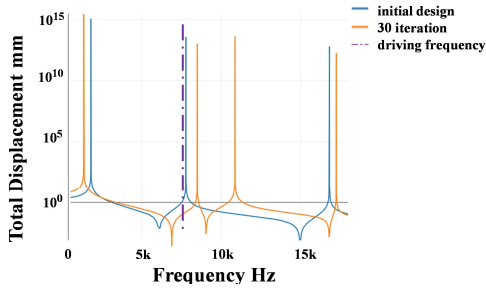

d)

Fig. 3. Optimization history: a) frequency, b) displacement, c) topology history, d) FRF

\section{A brief comparison with existing ESO and BESO methods}

Xie and Steven used a hard-kill ESO method for dynamics optimization problems in 1990s [10]. They calculated the sensitivity number for each element as follows:

$\alpha_{i}=\frac{1}{m_{i}}\left\{u_{i}^{e}\right\}^{T}\left(\omega_{i}^{2}\left[M^{e}\right]-\left[K^{e}\right]\right)\left\{u_{i}^{e}\right\}$

where $\alpha_{i}, m_{i}, u_{i}^{e}, \omega_{i}$ are the elemental sensitivity number, the modal mass, the elemental eigenvector and the corresponding natural frequency. $M^{e}$ and $K^{e}$ are the elemental mass matrix and the elemental stiffness matrix [10].

In order to avoid the direct deletion of elements, Huang has developed a new soft-kill BESO method for dynamics optimization problems in 2010 [11]. The sensitivity number is expressed as follows:

$\alpha_{i}=\left\{\begin{array}{l}\frac{1}{2 \omega_{i}}\left\{u_{i}^{e}\right\}^{T}\left(\frac{1-x_{\min }}{1-x_{\min }^{p}} K_{i}^{1}-\frac{\omega_{i}^{2}}{p} M_{i}^{1}\right)\left\{u_{i}^{e}\right\}, x_{i}=1, \\ \frac{1}{2 \omega_{i}}\left\{u_{i}^{e}\right\}^{T}\left(\frac{x_{\min }^{p-1}-x_{\min }^{p}}{1-x_{\min }^{p}} K_{i}^{1}-\frac{\omega_{i}^{2}}{p} M_{i}^{1}\right)\left\{u_{i}^{e}\right\}, \quad x_{i}=x_{\min },\end{array}\right.$

where $\omega_{i}$ and $u_{i}^{e}$ are the same as in Eq. (4). $x_{i}, x_{\min }$ are the elemental density and the lower limit of the density, $p$ is the penalty factor, $K_{i}^{1}$ and $M_{i}^{1}$ are the elemental stiffness matrix and the elemental mass matrix for the stiffer element [11].

As shown in Eqs. (4), (5), the sensitivity number of these two existing methods depends on the elemental mass matrix and the elemental stiffness matrix, which means that the calculation of the sensitivity number is much more expensive than in this paper, and it takes place outside the 
commercial FEM software.

Furthermore, these existing methods can only directly use the natural frequency as the optimization target. To the best of my knowledge, there is no existing ESO or BESO method concerning directly about the vibration reduction at a use-defined point of structures under a harmonic loading, while the SSDBM proposed in this paper use the vibration amplitude at a user-defined point directly as the optimization target. Additionally, the NFBM and the SSDBM take the influence of the load position into account at the same time.

\section{Conclusions}

This paper presents the von Mises stress as a sensitivity number and a new filter scheme for the BESO method focusing on dynamics topology optimization problems. The new filter scheme takes the static analysis, dynamic analysis and load position into account at the same time. Based on the extended BESO method, we propose two measures to reduce the vibration and demonstrate them with two numerical examples. Examples have shown that these two measures are effective for structures subjected to a single external harmonic loading, and that the vibration reduction effect of the SSDBM is better than the NFBM. Furthermore, the SSDBM does not need the MAC mode tracking technique, which saves computation time compared to the NFBM.

The calculation of the von Mises stress is not computationally expensive and takes place within the commercial FEA software. Furthermore, the BESO method reduces the FEM problem size after every iteration. Because of these advantages, these two proposed measures are very efficient from the aspect of computation time. We will extend these two measures in the future research, in order to make it suitable for large-scale FEM-meshes.

\section{Acknowledgements}

The authors would like to thank the German Research Foundation (DFG) for supporting this research under Grant No. BR2905/57-3: "Optimale Positionierung und Auslegung von Mehrmassendämpfern innerhalb eines kombinierten Topologieoptimierungsverfahrens".

\section{References}

[1] Huang X., Xie Y. M. Evolutionary Topology Optimization of Continuum Structures: Methods and Applications. John Wiley and Sons, 2010.

[2] Zhang W. H., Zhu J.H., Gao T. Topology Optimization in Engineering Structure Design. ISTE Press - Elsevier, 2016.

[3] Bendsøe M. P., Sigmund O. Topology Optimization Theory. Theory, Methods and Applications, Springer-Verlag Berlin Heidelberg, 2003.

[4] Xie Y. M., Steven G. P. A simple evolutionary procedure for structural optimization. Computers and Structures, Vol. 49, Issue 5, 1993, p. 885-896.

[5] Yang X., Xie Y. M., Steven G. P., Querin O. M. Bidirectional evolutionary method for stiffness optimization. AIAA Journal, Vol. 37, Issue 11, 1999, p. 1483-1488.

[6] Huang X., Xie Y. M. Convergent and mesh-independent solutions for the bi-directional evolutionary structural optimization method. Finite Elements in Analysis and Design, Vol. 43, Issue 14, 2007, p. 1039-1049.

[7] Jong C., Springer W. Teaching von Mises stress: from principal axes to nonprincipal axes. Annual Conference and Exposition, Austin, Texas, 2009.

[8] Querin O. M., Young V., Steven G., Xie Y. M. Computational efficiency and validation of bidirectional evolutionary structural optimization. Computer Methods in Applied Mechanics and Engineering, Vol. 189, Issue 2, 2000, p. 559-573.

[9] Patora M., Binda M. Modal assurance criterion. Procedia Engineering, Vol. 48, 2012, p. 543-548.

[10] Xie Y. M., Steven G. P. Evolutionary structural optimization for dynamic problems. Computers and Structures, Vol. 58, Issues 6-17, 1996, p. 1067-1073.

[11] Huang X., Zuo Z.H., Xie Y. M. Evolutionary topological optimization of vibrating continuum structures for natural frequencies. Computers and Structures, Vol. 88, Issues 5-6, 2010, p. 357-364. 